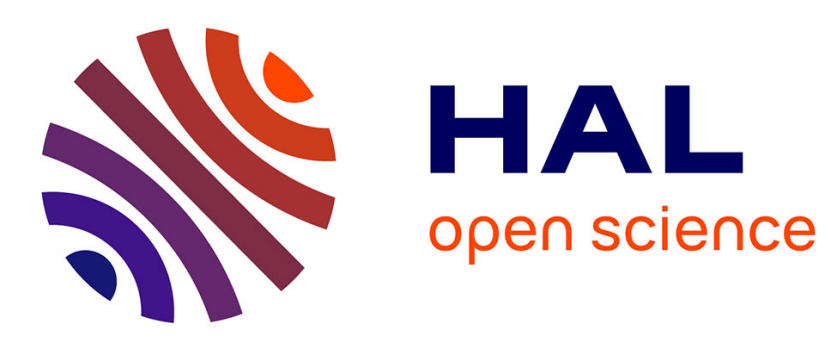

\title{
Near-Field and Far-Field Thermal Emission of an Individual Patch Nanoantenna
}

Claire Li, Valentina Krachmalnicoff, Patrick Bouchon, Julien Jaeck, Nathalie Bardou, Riad Haidar, Yannick de Wilde

\section{- To cite this version:}

Claire Li, Valentina Krachmalnicoff, Patrick Bouchon, Julien Jaeck, Nathalie Bardou, et al.. NearField and Far-Field Thermal Emission of an Individual Patch Nanoantenna. Physical Review Letters, 2018, 121, pp.243901-1 - 243901-6. 10.1103/PhysRevLett.121.243901 . hal-02055698

\section{HAL Id: hal-02055698 https://hal.science/hal-02055698}

Submitted on 4 Mar 2019

HAL is a multi-disciplinary open access archive for the deposit and dissemination of scientific research documents, whether they are published or not. The documents may come from teaching and research institutions in France or abroad, or from public or private research centers.
L'archive ouverte pluridisciplinaire HAL, est destinée au dépôt et à la diffusion de documents scientifiques de niveau recherche, publiés ou non, émanant des établissements d'enseignement et de recherche français ou étrangers, des laboratoires publics ou privés. 


\title{
Near-Field and Far-Field Thermal Emission of an Individual Patch Nanoantenna
}

\author{
Claire Li, ${ }^{1,2}$ Valentina Krachmalnicoff, ${ }^{1}$ Patrick Bouchon, ${ }^{2}$ Julien \\ Jaeck, ${ }^{2}$ Nathalie Bardou, ${ }^{3}$ Riad Haïdar, ${ }^{2, *}$ and Yannick De Wilde ${ }^{1, \dagger}$ \\ ${ }^{1}$ ESPCI Paris, PSL Research University, CNRS, Institut Langevin, 1 rue Jussieu, F-75005 Paris, France \\ ${ }^{2}$ DOTA, ONERA, Université Paris-Saclay, F-91123 Palaiseau, France \\ ${ }^{3}$ Centre de Nanosciences et de Nanotechnologies, CNRS, Université Paris-Sud, \\ Université Paris-Saclay, avenue de la Vauve, F-91120 Palaiseau, France
}

(Dated: November 13, 2018)

\begin{abstract}
The far-field spectral and near-field spatial responses of an individual metal-insulator-metal nanoantenna are reported, using thermal fluctuations as an internal source of electromagnetic field. The far-field spectra, obtained by combining Fourier transform infrared spectroscopy with spatial modulation based on a light falloff effect in a confocal geometry, have revealed two distinct emission peaks attributed to the excitation of the fundamental mode of the nanoantenna at two distinct wavelengths. Super-resolved near-field images of the thermally excited mode have been obtained by thermal radiation scanning tunneling microscopy. Experimental results are supported by numerical simulations showing that it is possible to excite the same mode at different wavelengths near a resonance of the insulating dielectric material forming the antenna.

PACS numbers: 44.40.+a, 68.37.Uv, 42.70.Km
\end{abstract}

Controlling the emission and absorption properties of surfaces and finite sized objects at infrared (IR) wavelengths is important for applications such as radiative cooling [1], thermal extraction [2,3], designing absorbers for detection [4-8], molecular sensing [9] or thermophotovoltaic energy conversion [10]. Another realm of interest is the production of novel IR sources with innovative features: spatial or spectral selectivity [11-14], subwavelength (sub- $\lambda$ ) dimensions [15-17] and modulation capabilities $[18,19]$. While thermal sources with no particular surface structuration are quasi-isotropic and spectrally broad in the far field, the surface patterning of materials with surface polaritons results in narrow diffraction lobes $[14,20]$, and, conversely, makes them perfectly absorbing for waves with a given incidence angle and wavelength [21]. Similar thermal antenna effects can be achieved over a broad spectral band with thin films structures [22]. Another promising way to achieve spectral [11-13] and angular [23, 24] control of thermal emission is through metasurfaces.

Thermal sources based on metasurfaces made of sub- $\lambda$ resonators generally involve the periodization of a unit cell containing one or several nanoantennas into an array. Diffraction as well as coupling between neighboring antennas are generally superimposed on the intrinsic optical response of the unit cell [25]. Measurements on nanoantennas ensembles also suffer from inhomogeneous broadening and statistical effects. Although it is essential to characterize a metasurface down to the unit cell level to provide precise knowledge of the intrinsic optical response, it remains challenging due to the sub- $\lambda$ dimensions of the object which call for high spatial and spectral resolutions in conjunction with enough detection sensitivity to address an individual nanoantenna.

In this letter, we demonstrate experimentally emission spectrum measurement and super-resolved mapping of a single nanoantenna, sub- $\lambda$ in the infrared spectral range of interest $(6-12 \mu \mathrm{m})$, based on the detection of its thermal radiation. Using far-field spectroscopy and thermal radiation scanning tun- neling microscopy (TRSTM) [26], we reveal both the spectral resonances of an individual metal-insulator-metal (MIM) resonator excited by thermal fluctuations and the spatial distribution of the local density of states (LDOS) at sub- $\lambda$ scale. A spatial modulation spectroscopy method is proposed to extract the far-field thermal radiation of a sub- $\lambda$ MIM resonator from the huge and spatially extended background thermal radiation. A comparative analysis is performed between experimental results on MIM nanoantennas and numerical simulations. Usually for non-dispersive insulators, only one fundamental resonance is expected but here we show that we find multiple solutions.

The investigated MIM nanoantennas are nanoresonators composed of a stack of a $200 \mathrm{~nm}$-thick gold layer, a $180 \mathrm{~nm}$ thick $\mathrm{SiO}_{2}$ layer and a $50 \mathrm{~nm}$-thick gold patch of sub- $\lambda$ width $w$ as depicted in Fig. 1(a). They sustain Fabry-Perot resonances for the gap plasmon trapped below the patch, giving rise to strong local field enhancement as illustrated by the finite-domain time-difference (FDTD) calculations (Lumerical Solutions) of the electric field intensity $|E|^{2}$ at resonance in Fig. 1(b). The phase-matching condition ruling the resonance wavelengths $\lambda_{\text {res }}$ is given by [27]:

$$
\lambda_{\text {res }}=\frac{2 n_{\mathrm{eff}} w}{m-\frac{\phi_{r}}{\pi}}
$$

with $n_{\text {eff }}$ the real part of the effective index $\tilde{n}_{\text {eff }}=n_{\text {eff }}+i k_{\text {eff }}$ of the mode of a sub- $\lambda$ metallic waveguide [28], $m$ the mode order and $\phi_{r}$ a phase correction factor. The geometrydependant resonance enables straightforward tunability for a wide spectral range [29-32]. Based on Eq. 1, we tune the fundamental resonant wavelength $(m=1)$ around $\lambda_{\text {res }}=$ $7 \mu \mathrm{m}$ where $n_{\text {eff }}$ varies smoothly by changing the nanoantennas widths $w$ from $2 \mu \mathrm{m}$ to $2.4 \mu \mathrm{m}$. Square-shaped patches are chosen to realize narrowband omnidirectional unpolarized emitters in the mid-IR [5]. A scanning electron microscope 
(a)

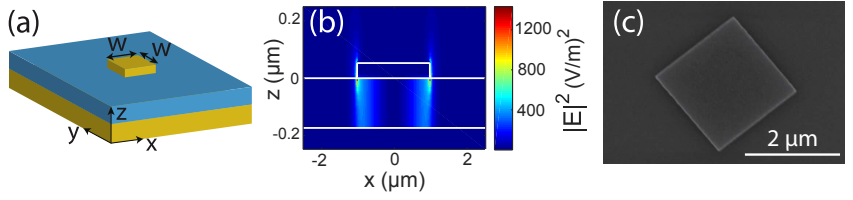

Figure 1. (color online). (a) Schematic of a MIM nanoantenna. (b) Calculated intensity of a nanoantenna at $y=0$ at resonance. (c) SEM image.

(SEM) image of one of the fabricated nanoantenna is depicted in Fig. 1(c).

Unlike previously reported investigations [33-35], the studies of individual nanoantennas presented here use the thermal emission of the sample as an internal source of electromagnetic field. It finds its origin in fluctuating thermal currents that excite both propagating and evanescent modes over a wide range of wavelengths, allowing to perform near-field and far-field measurements. While other studies of thermal emission from nanostructures rely either on periodic arrays [13] or on extended nanostructures in one direction [15], the technique we introduce is sensitive enough to extract the radiation of a nanoantenna that is both isolated and sub- $\lambda$ in all directions. To investigate the far-field thermal radiation spectrum of an individual IR nanoantenna, a major difficulty arises from the necessity to eliminate the dominant background thermal radiation. We bypass this issue by means of a spatial modulation spectroscopy method inspired from reported measurements at visible or near-IR wavelengths of the extinction by a single nanostructure spatially modulated in the Gaussian intensity profile of a focused laser beam [36-38]. However, in our case, the background thermal radiation is spatially extended around the MIM nanoantenna, thus requiring the introduction of a new paradigm to apply spatial modulation. Here, we take advantage instead of the dependence of the detected far-field thermal radiation signal with respect to the position of the nanoantenna projection on a detector in a confocal geometry while the background thermal radiation is spatially independent.

The spectroscopy set-up based on spatial modulation is shown in Fig. 2(a). A FTIR is placed in the infinity region of an IR microscope. The sample is heated at temperature $T_{\text {sample }}$ with a heating plate. The microscope uses a Cassegrain objective to collect the thermal radiation from the sample surface. A zinc selenide ( $\mathrm{ZnSe}$ ) lens at the exit of the FTIR spectrometer focuses the collected thermal radiation on the active area of a nitrogen-cooled mercury cadmium telluride (MCT) IR detector whose small size $\left(250 \times 250 \mu \mathrm{m}^{2}\right)$ produces a spatial selection. The IR detector captures the radiation from a $30 \mu \mathrm{m}$-sized region of the sample, hereafter called "confocal region". The IR thermal radiation from a single nanoantenna produces a spot in the image focal plane of the $\mathrm{ZnSe}$ lens with a diameter smaller but comparable with the size of the detector active area. The sample and the heating plate are installed on a piezoelectric translation stage allowing fine control of the nanoantenna position in the $x y$-plane. The thermal radiation signal from a single nanoantenna is practically undetectable as it is superimposed with a much more intense and spectrally distinct background thermal radiation signal which acts as a spectrally dependent offset. The emission cross-section of a 2 $\mu \mathrm{m}$-wide nanoantenna is calculated by FDTD numerical simulations to be $18 \mu \mathrm{m}^{2}$ at resonance which is only a few percent of the confocal region area. The power emitted by the nanoantenna scales with its emission cross-section. The fraction radiated towards the Cassegrain objective is typically $\sim$ $1 \mathrm{nW}$ and represents at maximum about $5 \%$ of of the thermal background as detailed in the Supplementary Material [39].

However, only the signal from the single nanoantenna exhibits a spatial dependence with respect to translations of the sample in the $x y$-plane while the background is spatially uniform. In fact, the signal coming from the nanoantenna is maximum when the latter is perfectly at the center of the confocal region and monotonously decays when its position shifts towards the edges, following a behavior which can be approximated by a Gaussian. This light fall-off effect of the signal from the nanoantenna as a function of its position is the keystone of the spatial modulation method that we have developed to extract the sole nanoantenna contribution from the thermal radiation signal measured by the IR detector in spite of the huge and dominant background.

We use a function generator to drive the piezoelectric stage with a sine modulation at frequency $\Omega$, displacing the sample periodically with an amplitude of a few micrometers along $x$. The spatial modulation of the MIM nanoantenna in the confocal region induces a small modulation of the intensity received by the IR detector. The lock-in demodulation at $\Omega$
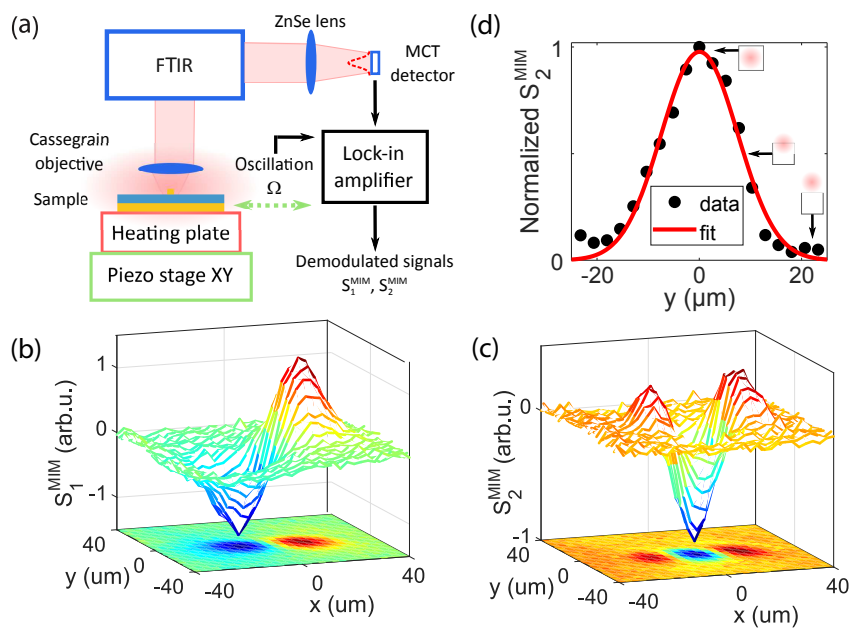

Figure 2. (color online). (a) Schematic of the spatial modulation spectroscopy set-up. (b-c) Three-dimensional plots of the raster scan of a spatially modulated nanoantenna in the $x$-direction, highlighting the spatial dependence of signal amplitude with respect to the nanoantenna position in the sample plane. The two-dimensional projection is represented in the $x y$-plane at the bottom. (d) Line section of the normalized central lobe of $S_{2}^{\mathrm{MIM}}$ along $y$ (black data points) and Gaussian fit (red line), showing the signal decay as the nanoantenna is displaced from the center toward the edges of the confocal region. 
and $2 \Omega$ is thus expected to suppress the spatially independent background, allowing to retrieve the first and second harmonics of the thermal radiation of the sole nanoantenna, $S_{1}^{\mathrm{MIM}}$ and $S_{2}^{\mathrm{MIM}}$ respectively. These two quantities are integrated over the spectral range of the IR detector. Their magnitude depends on the mean nanoantenna position with respect to the confocal region and also on the nanoantenna effective emission cross-section.

The spatial variations of $S_{1}^{\mathrm{MIM}}$ and $S_{2}^{\mathrm{MIM}}$ measured when raster scanning the nanoantenna in the $x y$-plane are presented in Fig. 2(b) and (c). Due to the expected behavior of the signal emitted by the single nanoantenna within the confocal region, $S_{1}^{\mathrm{MIM}}$ and $S_{2}^{\mathrm{MIM}}$ qualitatively reflect the spatial dependencies of the first and second derivative of a Gaussian-like function with respect to the direction of modulation $x$. The measured images of $S_{1}^{\mathrm{MIM}}$ and $S_{2}^{\mathrm{MIM}}$ show lobes of alternating signs in the $x$-direction.

The nanoantenna is at the center of the confocal region, i.e. optically conjugated with the center of the IR detector active region when $S_{1}^{\mathrm{MIM}}$ vanishes between the two lobes in Fig. 2(b) while at the same time $S_{2}^{\mathrm{MIM}}$ exhibits a pronounced extremum in Fig. 2(c). This enables fine positioning of the MIM antenna with sub- $\lambda$ accuracy within the confocal region despite being based on a purely far-field and therefore diffraction-limited optical measurement (see Supplemental Material [39]). Note that a cross-section through a lobe of $S_{1}^{\mathrm{MIM}}$ or $S_{2}^{\mathrm{MIM}}$ along the unmodulated direction $y$ directly reflects the behavior of the detector response to a sub- $\lambda$ source. This quantity, represented in Fig. 2(d) together with a Gaussian fit whose full width at half maximum is $17 \mu \mathrm{m}$, would not be accessible without modulation due to the background thermal radiation. We emphasize that the spatial variations reported here are similar to the ones observed with spatial modulation of a nano-object in a focused laser beam [36-38], but in our case they reflect the light fall-off effect of a sub- $\lambda$-sized source of thermal radiation within the confocal region.

The spectral measurements of the far-field thermal radiation of a nanoantenna at $T_{\text {sample }}=433 \mathrm{~K}$ were carried out by positionning it at an extremum of $S_{1}^{\mathrm{MIM}}$ to obtain maximum lock-in signal. We operate the FTIR in step scan mode [44] and the Fourier transform of the interferogram of $S_{1}^{\mathrm{MIM}}$ yields a spectrum that we correct from the instrumental response (i.e. detector sensitivity, optics transmission, absorption) by dividing it with the spectrum of a blackbody at $T_{\text {sample }}$ measured by the set-up with a chopper. Emission spectra measurements performed on single MIM nanoantennas for different widths $w$ and normalized to unity are depicted in Fig. 3(a). As expected, a resonant peak is observed at $\lambda_{\text {res }} \simeq 7 \mu \mathrm{m}$ but another is also present at $\simeq 11 \mu \mathrm{m}$ with both resonances exhibiting a red-shift with $w$.

The effective emission cross-section $\sigma_{e m}$ for MIM nanoantennas of varying widths $w$ are calculated by FDTD simulations, normalized to unity and depicted in Fig. 3(b). Two resonances are found with a good agreement with the experimental spectra. A small shift of $\sim 100 \mathrm{~nm}$ in the position of the two resonances is observed between the measured and simulated
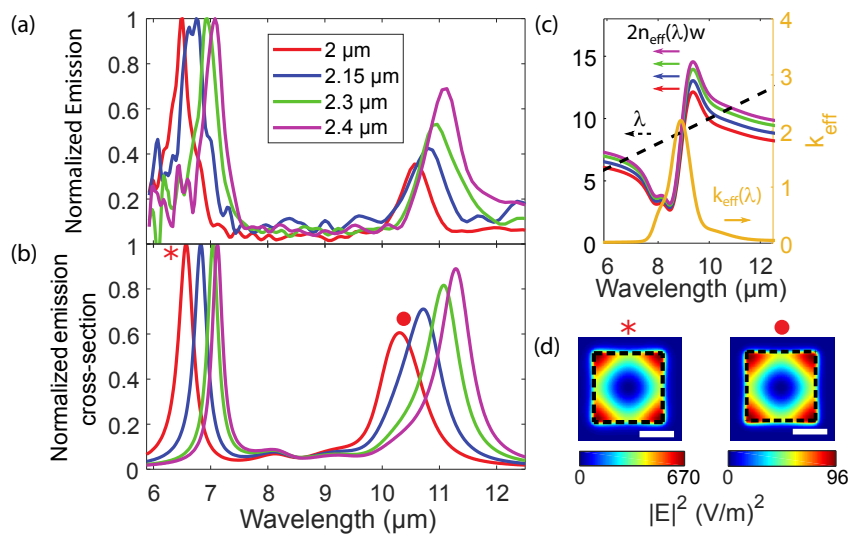

Figure 3. (color online). (a) Experimental emission spectra for a single nanoantenna for different widths $w$. (b) Calculated effective emission cross-sections. (c) Phase-matching condition for the fundamental mode $2 n_{\text {eff }}(\lambda) w=\lambda$ and effective extinction coefficient $k_{\text {eff }}$. (d) Calculated intensity maps inside the cavity of a MIM nanoantenna (dashed outline) for the two resonances denoted by the markers in (b). The white scale bars correspond to $1 \mu \mathrm{m}$.

spectra. The differences are attributed to the accuracy of the electron beam lithography used to pattern the square patches which produces errors of tens of nanometers on their nominal widths as verified by scanning electron microscopy. Another source of discrepancy is the robustness of the material models used in simulations (see Supplemental Material [39]) with the real and imaginary parts of the refractive index affecting the resonance position and amplitude respectively.

Consequently, both experimental and calculated emission spectra of the investigated MIM antennas exhibit not only one narrowband peak near $7 \mu \mathrm{m}$ but also another resonance near $11 \mu \mathrm{m}$. The latter is an unexpected result which cannot be ascribed to an harmonic of the fundamental mode. It originates from the strong variations of the permittivity of $\mathrm{SiO}_{2}$ in the spectral range between $8 \mu \mathrm{m}$ and $12 \mu \mathrm{m}$ thus affecting the Fabry-Perot resonance conditions for the gap plasmon. We illustrate in Fig. 3(c) the phase-matching condition for the fundamental mode by plotting $2 n_{\mathrm{eff}}(\lambda) w$ for various $w$. As $\phi_{r}$ is small enough to be neglected, we have $2 n_{\mathrm{eff}}(\lambda) w=\lambda$ according to Eq. 1. For a given value of $w$, three intersections with the dashed line $\lambda$ are found which correspond to the solutions $\lambda_{\text {res }}$. Only two resonances are observed experimentally and theoretically in Figs. 3(a) and 3(b). The third resonance near $9.5 \mu \mathrm{m}$ is suppressed because it is concomitant with strong absorption, as shown by the pronounced maximum of $k_{\text {eff }}$ plotted in Fig. 3(c). These material losses hinder the optical confinement inside the nanoantenna cavity at resonance. Indeed, as they increase, the peak emission decreases and broadens until it is completely suppressed, which is typical for a Fabry-Perot with intracavity losses [45]. Figure 3(d) displays the spatial maps of the mode distribution under the patch calculated at the two resonances. They exhibit an identical spatial distribution and confirm that the fundamental mode of the MIM nanoantenna is excited at both wavelengths. 
Remarkably, we have also been able to get an insight about the spatial distribution of the fundamental mode at sub- $\lambda$ scale by mapping the near-field thermal radiation at the surface of a MIM nanoantenna with the scattering tip of a TRSTM. The instrument relies on the thermal excitation of the electromagnetic modes and on the direct relation which exists between the local electromagnetic energy density and the LDOS $[26,46]$, a quantity which is not directly accessible with nearfield probes using either directional external sources to excite the electromagnetic modes of the sample [33,34] or thermovoltage measurements induced by a heat flux [47]. Note that the MIM antenna geometry is unfavorable for near-field measurements with a TRSTM. The latter is a surface probe while most of the electromagnetic energy of the thermally excited modes in a MIM nanoantenna is confined under the gold patch in a buried region inaccessible to the probe (Fig. 1(b)). This situation is dramatically different from other studies aimed at probing thermally excited surface polaritons at the interface between a material and air [26, 44, 48-50], and has limited us here to the realization of wavelength-integrated measurements.

The isothermal surface of the heated sample radiates a field whose local electromagnetic energy density is the LDOS multiplied by the average energy of a quantum oscillator [26, 44, 46, 48-51]. The TRSTM uses a tungsten tip oscillating at a frequency $\Omega_{\text {tip }}$ in intermittent contact with the sample along the $z$-direction and scatters the thermal radiation towards a mid-IR detector. A lock-in amplifier extracts the near-field thermal radiation signal by demodulating the detector signal at $\Omega_{\text {tip }}$ or a higher harmonic, yielding a signal $S_{n}^{\mathrm{NF}}$ with $n$ being the harmonic order [46]. The sample is raster scanned in the $x y$-plane and the recording of $S_{n}^{\mathrm{NF}}$ during the scan yields a super-resolved image with a spatial resolution $\sim$ $100 \mathrm{~nm}$ given by the curvature radius of the tip. The near-field optical image of $S_{2}^{\mathrm{NF}}$ using the full spectral width of the IR detector for a $2.4 \mu \mathrm{m}$-wide MIM nanoantenna heated at $403 \mathrm{~K}$ is represented in Fig. 4(a). As the two resonances observed in the far-field spectrum (Fig. 3(a-b)) share the same mode pattern, it enables signal enhancement for wavelength-integrated near-field imaging without having distinct modes overlapping in the resulting image.

The energy confined in the cavity formed by the dielectric layer leaks out from the discontinuities at the edges of the patch, leading to a pattern related to the resonance mode profile. The line scans across the patch edges (Fig. 4(c)) show that the TRSTM signal reveals maxima in all four corners of the patch. The image of Fig. 4(a) presents a slight asymmetry with a higher signal at the upper-right corner of the patch due to a small tilt of the TRSTM tip direction with respect to the perpendicular direction to the sample surface $z$.

We emphasize that other scanning near-field probes using scattering-tip [13, 34] or photothermal induced resonance $[52,53]$ techniques combined with an external laser excitation have revealed similar hot spots on nanostructures. While these techniques benefit from high incident field intensity and wavelength selectivity provided by tunable midinfrared lasers, the near-field images they produce reflect the distribution of the modes excited for a given direction and polarization of the laser excitation, which is different from the thermal excitation of all the modes performed with the TRSTM. (a)

(c)

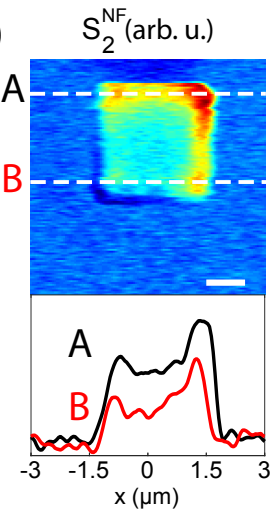

(b)

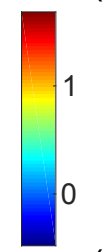

(d)
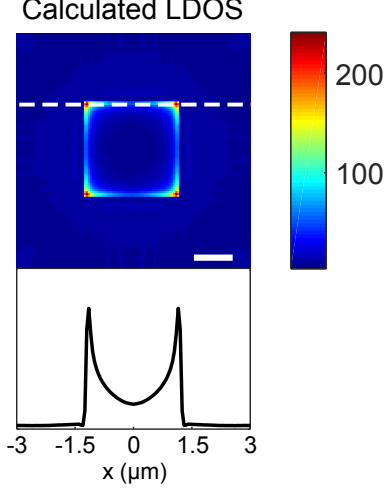

Figure 4. (color online). (a) Near-field optical distribution and (c) corresponding line scans across the top and bottom edges as indicated by the dashed lines. (b) Calculated LDOS map at resonance and (d) line scan across the edge. The white scale bars correspond to $1 \mu \mathrm{m}$.

For comparison with the experiments, FDTD simulations were performed to calculate the LDOS projected along $z$ which is the direction expected to give the highest TRSTM signal provided that the tip is aligned along $z$ [26]. The image (Fig. 4(b)) and corresponding line scan (Fig. 4(d)) are depicted for the first nanoantenna resonance. The calculated LDOS exhibits local maxima at the four corners of the square patch which is in good qualitative agreement with the TRSTM measurements of Figs. 4(a) and 4(c). The maps with sub$\lambda$ resolution provides useful information complementing the preceding spectral characterization. While the LDOS simulations shown in Fig. 4(b) and 4(d) are calculated without any tip, a detailed simulation of the TRSTM image would require to compute the signal radiated by the nanoantenna in the presence of the tungsten tip, whose position on the patch varies during the scan, and to take into account the demodulation process. The qualitative agreement observed between the calculated LDOS and the TRSTM maps suggests that the metallic tip of the TRSTM does not significantly perturb the near-field distribution around the MIM nanoantenna, except for the fact that it favors the detection along the $z$-axis. This may be due to the fact that it is scanned at the surface of the MIM nanoantenna, which does not disturb the region of high field confinement buried under the patch. However, a spectral shift of the resonances induced by the tip is not ruled out [44].

In summary, we have investigated the thermal radiation emitted by individual plasmonic nanoantennas. A method of spatial modulation has been implemented for background suppression in FTIR spectroscopy measurements of the far-field thermal radiation of sub- $\lambda$ sized objects, exploiting the spatial dependence of the detected signal which shows a light falloff effect in a confocal geometry. The far-field thermal radia- 
tion spectra measured on individual MIM nanoantennas have shown that the fundamental mode of a nanoantenna can be excited at distinct wavelengths around a resonance of the effective index of the plasmonic cavity. Using thermal radiation scanning tunneling microscopy, we have imaged the thermal near-fields at the surface of a MIM nanoantenna, and revealed the spatial distribution of its fundamental mode. The experimental methods used in this work can be readily applied to other nanostructures in the mid-IR. Notably, the FTIR spectroscopic approach combined with spatial modulation which we have developed could be used not only to retrieve the far-field thermal emission spectrum of a nanoantenna, but it could also be combined with an external broadband IR source for the farfield characterization of any kind of deeply sub-wavelength nanostructures or nanomaterials by FTIR spectroscopy.

This work received financial support from Labex WIFI (ANR-10-LABX-24 and ANR-10-IDEX-0001-02 PSL*) and ANR-16-CE09-0012 (CarISOVERRE). The authors are grateful to Karl Joulain (Institut Pprime) for enlightening discussions.

* Corresponding author: riad.haidar@onera.fr

${ }^{\dagger}$ Corresponding author: yannick.dewilde@espci.fr

[1] A. P. Raman, M. A. Anoma, L. Zhu, E. Rephaeli, and S. Fan, Nature 515, 540 (2014).

[2] Z. Yu, N. P. Sergeant, T. Skauli, G. Zhang, H. Wang, and S. Fan, Nat. Commun. 4, 1730 (2013).

[3] Y. Tan, B. Liu, S. Shen, and Z. Yu, Nanophotonics 5, 22 (2016).

[4] N. I. Landy, S. Sajuyigbe, J. J. Mock, D. R. Smith, and W. J. Padilla, Phys. Rev. Lett. 100, 207402 (2008).

[5] P. Bouchon, C. Koechlin, F. Pardo, R. Haïdar, and J.-L. Pelouard, Opt. Lett. 37, 1038 (2012).

[6] B. Fix, J. Jaeck, B. Vest, M. Verdun, G. Beaudoin, I. Sagnes, J.-L. Pelouard, and R. Haïdar, Appl. Phys. Lett. 111, 041102 (2017).

[7] Y. Nga Chen, Y. Todorov, B. Askenazi, A. Vasanelli, G. Biasiol, R. Colombelli, and C. Sirtori, Appl. Phys. Lett. 104, 031113 (2014).

[8] D. Palaferri, Y. Todorov, A. Bigioli, A. Mottaghizadeh, D. Gacemi, A. Calabrese, A. Vasanelli, L. Li, A. G. Davies, E. H. Linfield, et al., Nature 556, 85 (2018).

[9] H. Aouani, H. Sipova, M. Rahmani, M. Navarro-Cia, K. Hegnerova, J. Homola, M. Hong, and S. A. Maier, ACS Nano 7, 669 (2012).

[10] A. Lenert, D. M. Bierman, Y. Nam, W. R. Chan, I. Celanović, M. Soljačić, and E. N. Wang, Nat. Nanotechnol. 9, 2013 (2014).

[11] X. Liu, T. Tyler, T. Starr, A. F. Starr, N. M. Jokerst, and W. J. Padilla, Phys. Rev. Lett. 107, 045901 (2011).

[12] M. Makhsiyan, P. Bouchon, J. Jaeck, J.-L. Pelouard, and R. Haïdar, Appl. Phys. Lett. 107, 251103 (2015).

[13] T. Wang, P. Li, D. N. Chigrin, A. J. Giles, F. J. Bezares, O. J. Glembocki, J. D. Caldwell, and T. Taubner, ACS Photonics 4, 1753 (2017).

[14] J.-J. Greffet, R. Carminati, K. Joulain, J.-P. Mulet, S. Mainguy, and Y. Chen, Nature 416, 61 (2002).

[15] J. A. Schuller, T. Taubner, and M. L. Brongersma, Nat. Photonics 3, 658 (2009).
[16] T. Lewi, P. P. Iyer, N. A. Butakov, A. A. Mikhailovsky, and J. A. Schuller, Nano Lett. 15, 8188 (2015).

[17] K. Joulain, Y. Ezzahri, and R. Carminati, J. Quant. Spectrosc. Radiat. Transfer 173, 1 (2016).

[18] E. Sakat, L. Wojszvzyk, J.-P. Hugonin, M. Besbes, C. Sauvan, and J.-J. Greffet, Optica 5, 175 (2018).

[19] V. W. Brar, M. C. Sherrott, M. S. Jang, S. Kim, L. Kim, M. Choi, L. A. Sweatlock, and H. A. Atwater, Nat. Commun. 6, 7032 (2015).

[20] P. J. Hesketh, J. N. Zemel, and B. Gebhart, Nature 324, 549 (1986).

[21] M. Hutley and D. Maystre, Opt. Commun. 19, 431 (1976).

[22] P. Ben-Abdallah, J. Opt. Soc. Am. 21, 1368 (2004).

[23] W. Wang, C. Fu, and W. Tan, J. Quant. Spectrosc. Radiat. Transfer 132, 36 (2014).

[24] D. Costantini, A. Lefebvre, A.-L. Coutrot, I. Moldovan-Doyen, J.-P. Hugonin, S. Boutami, F. Marquier, H. Benisty, and J.-J. Greffet, Phys. Rev. A. 4, 014023 (2015).

[25] P. Chevalier, P. Bouchon, J. Jaeck, D. Lauwick, N. Bardou, A. Kattnig, F. Pardo, and R. Haïdar, Appl. Phys. Lett. 107, 251108 (2015).

[26] Y. De Wilde, F. Formanek, R. Carminati, B. Gralak, P.-A. Lemoine, K. Joulain, J.-P. Mulet, Y. Chen, and J.-J. Greffet, Nature 444, 740 (2006).

[27] J. Yang, C. Sauvan, A. Jouanin, S. Collin, J.-L. Pelouard, and P. Lalanne, Opt. Express 20, 16880 (2012).

[28] S. Collin, F. Pardo, and J.-L. Pelouard, Opt. Express 15, 4310 (2007).

[29] C. Belacel, B. Habert, F. Bigourdan, F. Marquier, J.-P. Hugonin, S. Michaelis de Vasconcellos, X. Lafosse, L. Coolen, C. Schwob, C. Javaux, B. Dubertret, J.-J. Greffet, P. Senellart, and A. Maitre, Nano Lett. 13, 1516 (2013).

[30] J. Hao, J. Wang, X. Liu, W. J. Padilla, L. Zhou, and M. Qiu, Appl. Phys. Lett. 96, 251104 (2010).

[31] N. I. Landy, C. M. Bingham, T. Tyler, N. Jokerst, D. R. Smith, and W. J. Padilla, Phys. Rev. B. 79, 125104 (2009).

[32] P. Jouy, Y. Todorov, A. Vasanelli, R. Colombelli, I. Sagnes, and C. Sirtori, Appl. Phys. Lett. 98, 021105 (2011).

[33] R. L. Olmon, M. Rang, P. M. Krenz, B. A. Lail, L. V. Saraf, G. D. Boreman, and M. B. Raschke, Phys. Rev. Lett. 105, 167403 (2010).

[34] P. Alonso-González, P. Albella, F. Neubrech, C. Huck, J. Chen, F. Golmar, F. Casanova, L. E. Hueso, A. Pucci, J. Aizpurua, and R. Hillenbrand, Phys. Rev. Lett. 110, 203902 (2013).

[35] F. Neubrech, A. Pucci, T. W. Cornelius, S. Karim, A. GarcíaEtxarri, and J. Aizpurua, Phys. Rev. Lett. 101, 157403 (2008).

[36] A. Arbouet, D. Christofilos, N. Del Fatti, F. Vallée, J. R. Huntzinger, L. Arnaud, P. Billaud, and M. Broyer, Phys. Rev. Lett. 93, 127401 (2004).

[37] O. L. Muskens, N. Del Fatti, F. Vallée, J. R. Huntzinger, P. Billaud, and M. Broyer, Appl. Phys. Lett. 88, 063109 (2006).

[38] M. Husnik, M. W. Klein, N. Feth, M. König, J. Niegemann, K. Busch, S. Linden, and M. Wegener, Nat. Photonics 2, 614 (2008).

[39] "See supplemental material at [url] for further details which include Refs. [40-43]".

[40] M. P. McDonald, F. Vietmeyer, D. Aleksiuk, and M. Kuno, Rev. Sci. Instrum. 84, 113104 (2013).

[41] C. Koechlin, P. Bouchon, F. Pardo, J. Jaeck, X. Lafosse, J.-L. Pelouard, and R. Haïdar, Appl. Phys. Lett. 99, 241104 (2011).

[42] J. Kischkat, S. Peters, B. Gruska, M. Semtsiv, M. Chashnikova, M. Klinkmüller, O. Fedosenko, S. Machulik, A. Aleksandrova, G. Monastyrskyi, et al., Appl. Opt. 51, 6789 (2012).

[43] R. Kitamura, L. Pilon, and M. Jonasz, Appl. Opt. 46, 8118 
(2007).

[44] A. Babuty, K. Joulain, P.-O. Chapuis, J.-J. Greffet, and Y. De Wilde, Phys. Rev. Lett. 110, 146103 (2013).

[45] D. Hofstetter and R. L. Thornton, Opt. Lett. 22, 1831 (1997).

[46] R. Carminati, A. Cazé, D. Cao, F. Peragut, V. Krachmalnicoff, R. Pierrat, and Y. De Wilde, Surf. Sci. Rep. 70, 1 (2015).

[47] A. Kittel, U. F. Wischnath, J. Welker, O. Huth, F. Rueting, and S.-A. Biehs, Appl. Phys. Lett. 93, 193109 (2008).

[48] Y. Kajihara, K. Kosaka, and S. Komiyama, Rev. Sci. Instrum. 81, 033706 (2010).
[49] F. Peragut, J.-B. Brubach, P. Roy, and Y. De Wilde, Appl. Phys. Lett. 104, 251118 (2014).

[50] A. C. Jones and M. B. Raschke, Nano Lett. 12, 1475 (2012).

[51] K. Joulain, R. Carminati, J.-P. Mulet, and J.-J. Greffet, Phys. Rev. B. 68, 245405 (2003).

[52] B. Lahiri, G. Holland, V. Aksyuk, and A. Centrone, Nano Lett. 13, 3218 (2013).

[53] E. Calandrini, T. Venanzi, F. Appugliese, M. Badioli, V. Giliberti, L. Baldassarre, P. Biagioni, F. De Angelis, W. M. Klesse, G. Scappucci, et al., Appl. Phys. Lett. 109, 121104 (2016). 